\title{
Supporting Information: \\ PCM-ROKS for the Description of Charge-Transfer States in Solution: Singlet-Triplet Gaps with Chemical Accuracy from Open-Shell Kohn-Sham Reaction-Field Calculations
}

\author{
Lukas Kunze, ${ }^{a}$ Andreas Hansen, ${ }^{a}$ Stefan Grimme, ${ }^{a}$ and Jan-Michael Mewes ${ }^{* a}$ \\ ${ }^{a}$ Mulliken Center for Theoretical Chemistry, Institut für Physikalische und Theoretische Chemie, \\ Rheinische Friedrich-Wilhelms Universität Bonn, Beringstraße 4, 53115 Bonn, Germany
}

(Dated: August 26, 2021)

\section{Computational Details and Workflow}

All quantum-chemical calculations were carried out with a development version of the Q-Chem 5.4 program package ${ }^{1}$ We have modified the original source code to enable ROKS optimizations in combination with the IEF-PCM solvent model. The respective modifications will be included in the next Q-Chem release (5.4.1). Single-point ROKS/IEF-PCM calculations, ROKS optimizations with C-PCM, and UKS/IEF-PCM optimizations should work in previous versions. All calculations and tuning protocols use the def2-SVP basis set. ${ }^{2}$

The molecular environment is represented using a polarizable-continuum model (PCM) with the integral equation formalism (IEF) ${ }^{3}$ and the Q-Chem default radii (Bondi*1.2). Parameters for toluene $(\varepsilon=2.37$, $\left.n^{2}=2.24\right)$ were chosen due to the widespread use in experiments relating TADF emitters and benchmark calculations. Additional calculations have been conducted with $\varepsilon=3.5$ (corresponding to the most polar host materials) to test how this affects the calculated gaps.

Three different RSH functionals were tested, namely, the CAM-QTP $(01)^{4}$, the LC- $\omega \mathrm{PBE}^{5}{ }^{17}$ and the $\omega \mathrm{B} 97 \mathrm{M}-$ $V^{8} \mathrm{RSH}$ functional. All three functionals recover $100 \%$ non-local exchange at large distances essential for the correct description of CT states. The CAM-QTP(01) functional is chosen since it was specifically fitted to reproduce Koopman's theorem aligning vertical ionization energies of the first four occupied orbitals of water. The LC- $\omega$ PBE functional was one of the first RSH functionals and showed a very good performance in benchmarks on excitation energies calculated by TD-DFT in combination with optimal tuning. In contrast to the other two functionals, $\omega \mathrm{B} 97 \mathrm{M}-\mathrm{V}$, is currently one of the most accurate all-purpose RSH meta-GGAs which was not explicitly designed for application in excitedstate chemistry. Additionally, $\mathrm{PBE}\left(\frac{9110}{}\right.$ employing the DFT-D $4^{11112}$ dispersion correction was tested to gauge the accuracy of the popular global hybrid compared to RSH functionals.

The D3 dispersion correction with the default BeckeJohnson damping and 3-body terms is applied for the
LC- $\omega$ PBE functional ${ }^{[1314}$ Note that we use a Q-Chem version in which a previous problem with the gradients of the D3-correction has been fixed. For the CAMQTP(01) functional, we do not use any dispersion correction due to a lack of parameters. Later in the study, we found a D3(BJ) parameterization by Jan M. L. Martin and coworkers, by this time however, the effect of the dispersion correction had already proven to be insignificant ${ }^{15} \omega \mathrm{B} 97 \mathrm{M}-\mathrm{V}$ incorporates London dispersion effects via the VV10 non-local Van-der-Waals functional! 16

In the context of UKS(IMOM) and ROKS many different initial guess orbitals were tested, including using converged ground-state orbitals, converged ROKS orbitals for UKS(IMOM) and converged orbitals of the cation of the respective molecule. Generally, ROKS converged much more reliably to the lowest singlet state than UKS(IMOM) when simple ground-state orbitals are provided. Therefore, we at first exploited this by giving UKS(IMOM) converged ROKS orbitals as initial guess, leading to robust convergence to the lowest singlet in the majority of cases. However, in the revision process we noted another viable option might be using converged orbitals from the cationic species of the molecule as input, which worked well in toluene environment but may possibly be problematic for more polar solvents. In the preceding cation single point calculation, the orbital ordering is chosen such that the vacant orbital in the cationic species gets filled with the excited electron in the subsequent UKS(IMOM) or ROKS calculation. Naturally, the preceding cation single point has to be chosen as an unrestricted open-shell or restricted open-shell calculation for $\triangle \mathrm{SCF}$ and ROKS, respectively. Generally, when calculating excited state via $\triangle \mathrm{SCF}$ one should definitely check different initial guess orbitals.

The same grid settings were used for all three functionals, namely a Lebedev grid with 70 radial grid and 590 angular points per atom. Intermediary investigations showed no need for large grid sizes, but the large grid was kept due to consistency and comparability reasons of the results. 


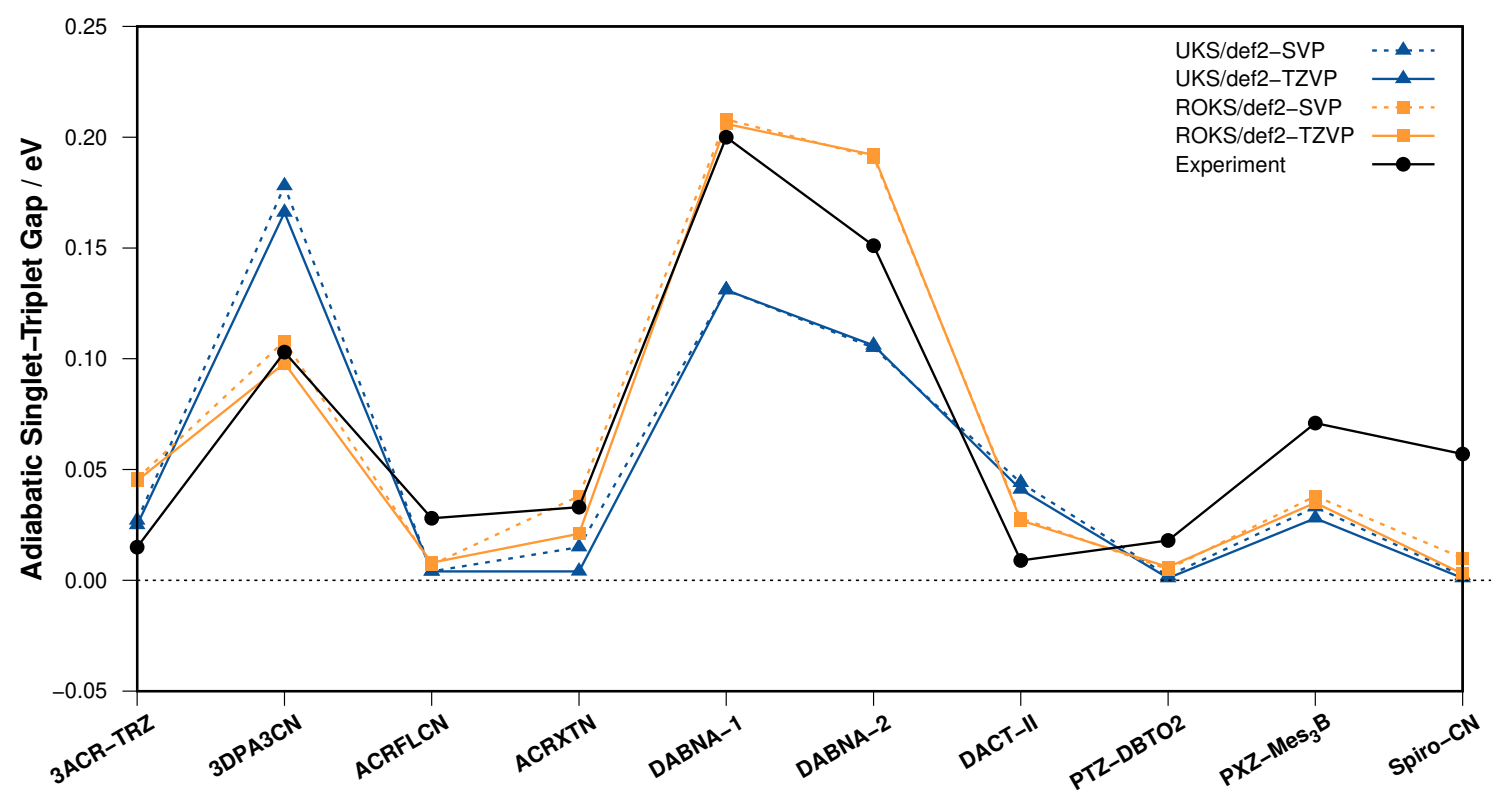

Figure S1. Comparison of $\Delta E_{\mathrm{ST}}$ calculated with the OT-LC- $\omega \mathbf{P B E}-\mathrm{D} 3$ functional by the UKS and ROKS method using the def2-SVP and def2-TZVP basis set, all values in $\mathrm{eV}$.

\section{Optimal Tuning of Range-Separated Hybrid Functionals}

A non-empirical, system-dependent procedure for determining the optimal $\omega$ value based on Koopman's theorem modified according to Head-Gordon and coworkers ${ }^{17}$ was applied. The physically sound approach connects HOMO energies of the neutral and single negatively charged molecule with their respective first ionization potentials by the target function

$$
J^{2}(\omega)=\sum_{N=Z, Z+1}\left[\epsilon_{N}^{H O M O}(\omega)-I P_{N}(\omega)\right]^{2}
$$

which yields the optimal $\omega$ value for its minimal value.

The idea of a fixed $\omega$ value was introduced very early in the work, and therefore does not include the whole set $\mathrm{A}$. The marginal accuracy difference in $\Delta E_{\mathrm{ST}}$ from using OT or fixed $\omega$ values for the whole set A shows clearly almost no loss in accuracy by not averaging over the whole set A. This might be due to this particular subset being relative balanced regarding emitter types. Additionally, due to emitter changes in the subset, the fixed $\omega$ values actually used in the calculations differ from the reported ones below, which has due to the insignificant change $(\Delta \omega=0.001)$ virtually no effect on the resulting gaps.
Table S1. Optimal tuning $\omega$ values and statistical parameters for all tested functionals for the preliminary subset of set A listed alphabetically, $\omega$ is dimensionless.

\begin{tabular}{llrrr}
\hline$\#$ & System & CAM-QTP(01) & $\omega$ B97M-V & LC- $\omega$ PBE-D3 \\
\hline 4 & PTZ-DBTO2 & 0.157 & 0.180 & 0.199 \\
5 & ACRXTN & 0.150 & 0.174 & 0.196 \\
7 & PXZ-Mes 3 B & 0.137 & 0.161 & 0.177 \\
10 DACT-II & 0.133 & 0.153 & 0.175 \\
17 3ACR-TRZ & 0.122 & 0.140 & 0.163 \\
21 3DPA3CN & 0.113 & 0.132 & 0.154 \\
24 & ACRFLCN & 0.140 & 0.160 & 0.181 \\
25 & Spiro-CN & 0.133 & 0.153 & 0.174 \\
26 DABNA-2 & 0.110 & 0.128 & 0.145 \\
27 DABNA-1 & 0.135 & 0.158 & 0.175 \\
\hline \multicolumn{2}{l}{ Mean } & $\mathbf{0 . 1 3 3}$ & $\mathbf{0 . 1 5 4}$ & $\mathbf{0 . 1 7 4}$ \\
\hline Min & 0.110 & 0.128 & 0.145 \\
Max & 0.157 & 0.180 & 0.199 \\
SD & 0.015 & 0.017 & 0.017 \\
\hline
\end{tabular}

\section{Basis Set Convergence}

The basis set convergence regarding the adiabatic singlet-triplet gap was investigated for the OT-LC$\omega \mathrm{PBE}-\mathrm{D} 3$ functional for a subset of set A of $10 \mathrm{TADF}$ emitters using the def2-SVP and def2-TZVP basis set, see figure S1. The observed differences between def2- 
SVP and def2-TZVP rarely exceed $0.010 \mathrm{eV}$, the MD for UKS/ROKS is $0.003 / 0.004 \mathrm{eV}$ while the MAD for $\mathrm{UKS} / \mathrm{ROKS}$ is $0.004 / 0.004 \mathrm{eV}$, respectively.

\section{Geometry Dependence of ROKS T1 Energies}

Table S2. Adiabatic $\Delta E_{\mathrm{ST}}$ by ROKS method with the LC- $\omega$ PBE-D3 functional, comparison of ROKS T1 energies calculated at the UKS geometry versus fully geometrically relaxed ROKS T1 energies, UKS and ROKS were denoted $\mathrm{U}$ and $\mathrm{RO}$ respectively, all values in $\mathrm{eV}$.

\begin{tabular}{|c|c|c|c|}
\hline$\#$ & System & $\mathrm{RO}(\mathrm{T} 1) @ \mathrm{U}(\mathrm{T} 1)$ & $\mathrm{RO}(\mathrm{T} 1) @ \mathrm{RO}(\mathrm{T} 1)$ \\
\hline 4 & PTZ-DBTO2 & 0.0047 & 0.0050 \\
\hline 5 & ACRXTN & 0.0377 & 0.0398 \\
\hline 7 & $\mathrm{PXZ}^{-\mathrm{Mes}_{3} \mathrm{~B}}$ & 0.0380 & 0.0382 \\
\hline 10 & DACT-II & 0.0276 & 0.0282 \\
\hline 17 & 3ACR-TRZ & 0.0462 & 0.0476 \\
\hline 21 & 3DPA3CN & 0.1077 & 0.1078 \\
\hline 24 & ACRFLCN & 0.0071 & 0.0074 \\
\hline 25 & Spiro-CN & -0.0068 & -0.0030 \\
\hline 26 & DABNA-2 & 0.1905 & 0.1908 \\
\hline 27 & DABNA-1 & 0.2082 & 0.2085 \\
\hline & $\mathrm{MD}$ & -0.0009 & - \\
\hline & MAD & 0.0009 & - \\
\hline & AMAX & 0.0021 & - \\
\hline
\end{tabular}

As described in this work, ROKS energies for the lowest triplet state (T1) were obtained by performing single point calculations on the lowest UKS-optimized triplet state, to save computation time. Table S2 shows the observed difference in $\Delta E_{\mathrm{ST}}$ for $\mathrm{ROKS}(\mathrm{T} 1)$ single point calculations on UKS(T1)-optimized geometries compared to fully geometrically relaxed ROKS(T1) energies. For this purpose, tests were carried out for a subset of set A consisting of 10 TADF emitters. The obtained results show a MAD of only $<0.001 \mathrm{eV}$ and a maximum absolute error of only $0.002 \mathrm{eV}$, thus validating the accuracy of the applied ROKS T1 single point calculation approach.

\section{Solvation Effects: Dielectric Constant Dependence}

The dependence of the adiabatic singlet-triplet gap on the environments dielectric constant was investigated with the OT-LC- $\omega$ PBE-D3 functional, comparing calculated gaps employing $\varepsilon=2.37$ (toluene) or $\varepsilon=3.5$ $(\approx \mathrm{PPF})$ as environment polarity. Five emitters are missing from this subset of the whole benchmark, since they were added later. Figure S2 shows that employing a more polar environment systematically decreases the calculated $\Delta E_{\mathrm{ST}}$. This is because the characters of the excited and triplet states align (become more CTlike) in more polar environments, In turn, those emitters (DABNA-1, DABNA-2, p-2Cz2BMe) where the lowest excited state was predicted to be of locally excited character display no changes or only minor changes in $\Delta E_{\mathrm{ST}}$.

Table S3. Statistical analysis of adiabatic $\Delta E_{\mathrm{ST}}$ calculated with the OT-LC- $\omega$ PBE-D3 by the UKS and ROKS method with a dielectric constant of $\varepsilon=3.5$, all values in $\mathrm{eV}$.

\begin{tabular}{lrrrrr}
\hline Method & MD & MAD & AMIN & AMAX & SD \\
\hline UKS & -0.020 & 0.027 & 0.002 & 0.071 & 0.027 \\
ROKS & -0.016 & 0.024 & 0.000 & 0.069 & 0.028 \\
\hline
\end{tabular}

\section{Effect of London Dispersion Correction DFT-D4}

The effect of London dispersion included via the D4 correction to the PBE0 functional on calculated singlettriplet gaps was investigated. A subset of set A was used for this purpose. As shown in figure S3 the dispersion correction has almost no effect on $\Delta E_{\mathrm{ST}}$. The only significant difference is visible for ACRFLCN, where the dispersion effects stabilize the bending of the spirocenter leading to different conformations for PBE0 and PBE0-D4, and ultimately to different lowest excited states.

Table S4. Statistical analysis of $\Delta E_{\mathrm{ST}}$ calculated with the PBE0 and PBE0-D4 functionals compared to experimental values and against each other, all values in $\mathrm{eV}$.

\begin{tabular}{|c|c|c|c|c|c|}
\hline Functional & Method & MD & MAD & AMIN & AMAX \\
\hline \multirow{2}{*}{ PBE0 vs. Exp. } & UKS & -0.013 & 0.041 & 0.006 & 0.122 \\
\hline & ROKS & 0.027 & 0.039 & 0.019 & 0.061 \\
\hline \multirow{2}{*}{ PBE0-D4 vs Exp. } & UKS & -0.015 & 0.039 & 0.004 & 0.122 \\
\hline & ROKS & 0.025 & 0.035 & 0.001 & 0.055 \\
\hline \multirow{2}{*}{ PBE0 vs. PBE0-D4 } & UKS & 0.002 & 0.003 & 0.000 & 0.022 \\
\hline & ROKS & 0.001 & 0.005 & 0.000 & 0.020 \\
\hline
\end{tabular}

\section{Timings of UKS, ROKS and TD-DFT Geometry Optimizations}

Table S5 summarizes the total walltime for an optimization step (last column) with each of the herein em- 


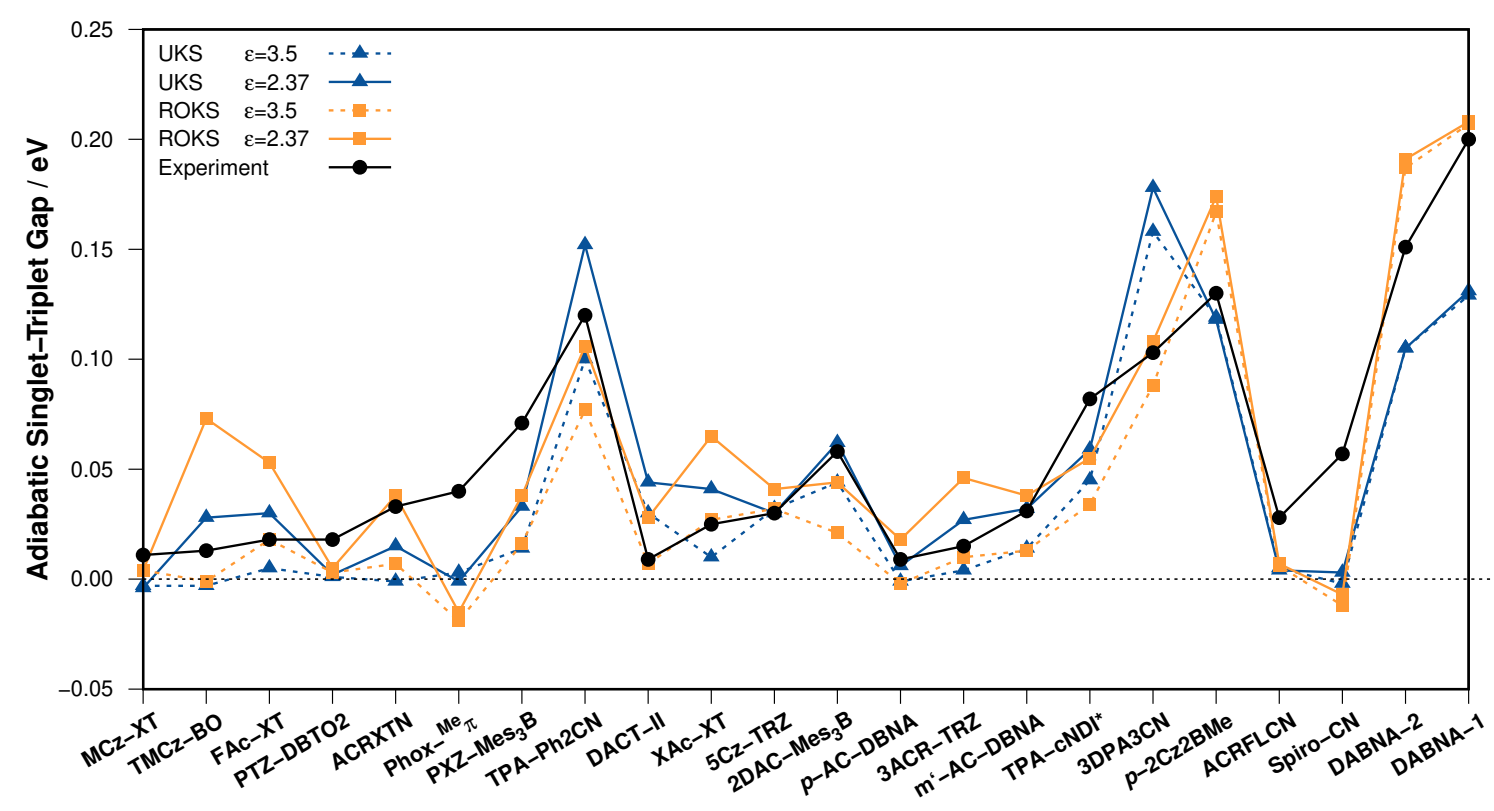

Figure S2. Comparison of $\Delta E_{\mathrm{ST}}$ calculated with the OT-LC- $\omega$ PBE-D3 functional by the UKS and ROKS method employing either $\varepsilon=2.37$ or $\varepsilon=3.5$ as dielectric constant, all values in $\mathrm{eV}$.

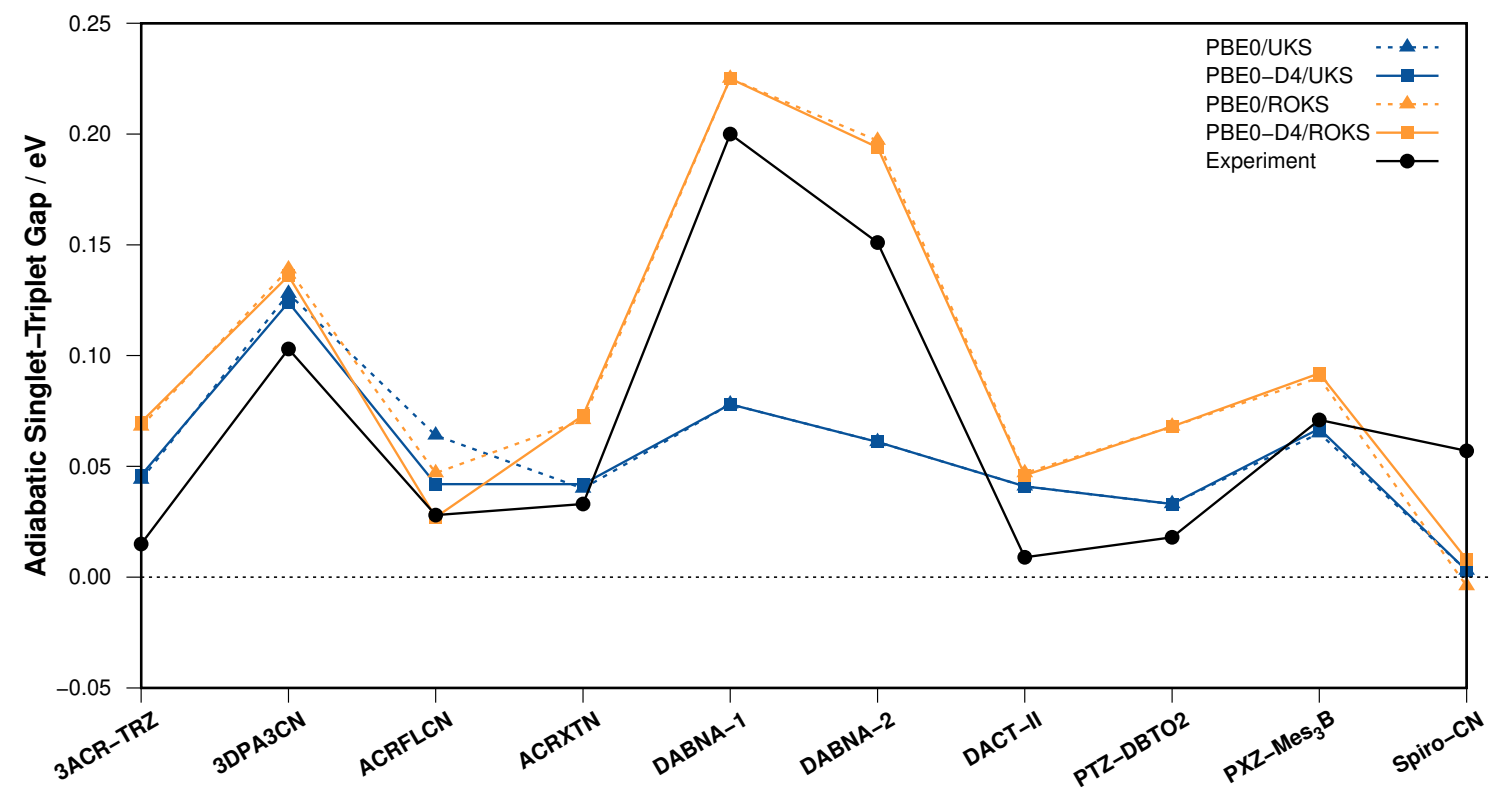

Figure S3. Comparison of $\Delta E_{\mathrm{ST}}$ calculated with the PBE0 and PBE0-D4 functionals by the UKS and ROKS method, all values in $\mathrm{eV}$.

ployed approaches for one of the smallest and largest TADF emitters of the set. These are TPA-Ph2Cn (\#8) with 237 contracted and 515 primitive basis functions, as well as 5Cz-TRZ (\#12) with 684 contracted and 1496 primitive functions (with def2-SVP). The considered integration grids are a highly accurate Lebedev grid with 70 radial and 590 angular grid points, as well as the
Q-Chem standard grid 1 (SG1), which is automatically assigned for the tested functional, FX-LC- $\omega$ PBE-D3. Timings are given for each step of the calculations, i.e., SCF, Gradient, and in case of TDA also the CI step. All calculations were carried out on 8 cores of a Intel(R) Xeon(R) CPU E5-2650 v2@ $@ 2.60 \mathrm{GHz}$ with 32 GB memory allocated. The provided timings are pro- 


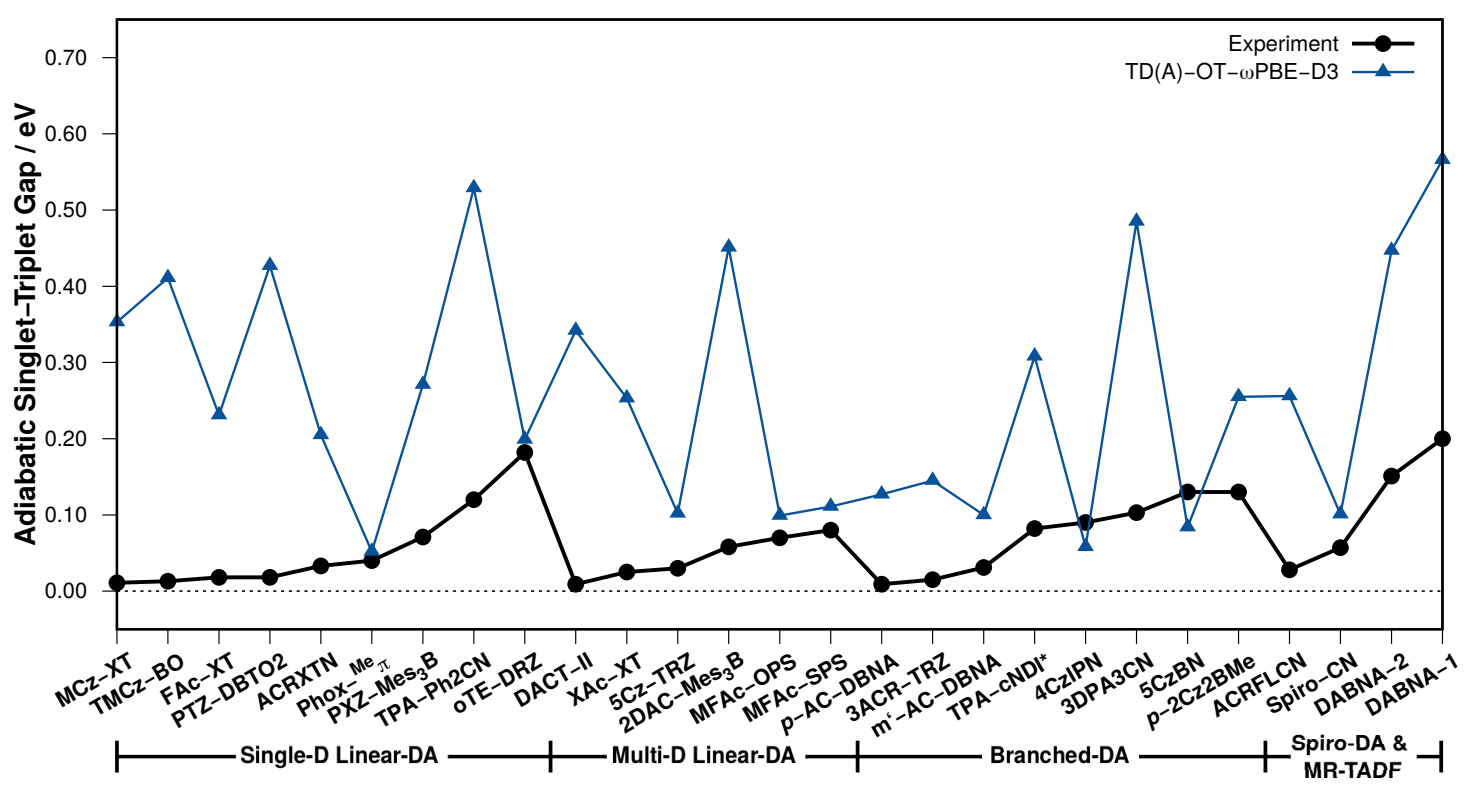

Figure S4. Adiabatic $\Delta E_{\mathrm{ST}}$ calculated with the OT-LC- $\omega$ PBE-D3 functional with TDA employing LR-PCM, all values in $\mathrm{eV}$.

Table S5. Walltimes (minutes) for one optimization step (averages over the first ten steps) with ROKS and UKS (both with PCM) compared to TD-DFT/LR-PCM. All in combination with the FX-LC- $\omega$ PBE-D3 functional and def2SVP basis for the TADF emitters 5Cz-TRZ (\#8) and TPAPh2CN (\#12).

\begin{tabular}{|c|c|c|c|c|c|c|}
\hline \multicolumn{7}{|c|}{ FX-LC- $\omega$ PBE-D3 } \\
\hline Mol. \# & Grid & Method & $\mathrm{SCF}$ & Grad. & CI & Tot. \\
\hline \multirow{6}{*}{8} & \multirow{3}{*}{ SG-1 } & ROKS & 15 & 2 & - & 17 \\
\hline & & UKS/MOM & 8 & 1 & - & 9 \\
\hline & & TDA & 4 & 4 & 16 & 24 \\
\hline & \multirow{3}{*}{70,590} & ROKS & 32 & 2 & - & 34 \\
\hline & & UKS/MOM & 14 & 1 & - & 15 \\
\hline & & TDA & 8 & 5 & 28 & 41 \\
\hline \multirow{6}{*}{12} & \multirow{3}{*}{ SG-1 } & ROKS & 255 & 20 & & 275 \\
\hline & & UKS/MOM & 84 & 10 & - & 94 \\
\hline & & TDA & 51 & 88 & 381 & 519 \\
\hline & \multirow{3}{*}{70,590} & ROKS & 338 & 30 & & 368 \\
\hline & & UKS/MOM & 113 & 15 & - & 128 \\
\hline & & TDA & 70 & 95 & 444 & 608 \\
\hline
\end{tabular}

gram internal printouts converted into minutes.

As evident from this comparison, the ROKS and in particular UKS optimizations are significantly faster (factor 1.4-4.8) under all circumstances than the respective TDA optimizations, in particular for the larger systems. This is despite significantly longer SCF timings of the open-shell UKS and ROKS approaches, which are overcompensated by the lack of the CI step (in which we include the calculation of relaxed densities) and faster gradient evaluation. Generally, UKS/MOM is the fastest method, followed by ROKS eventually TD/TDA-DFT.

\section{A. Adiabatic Gaps from TD(A)-DFT/LR-PCM Calculations}

For the sake of comparison, we also provide $\mathrm{TD}(\mathrm{A})$ $\omega \mathrm{PBE}-\mathrm{D} 3 / \mathrm{LR}-\mathrm{PCM}$ results for the full set. In analogy to the ROKS/UKS based workflow, we have obtained these gaps by optimizing the respective lowest excited singlet and triplet states at the TD(A)-DFT level. The results gaps much too large compared to experimental values since the dielectric stabilization of the singlet CT state is not correctly recovered by the LR-PCM model, causing a too large energy compared to the less polar (here mostly locally excited) triplet states.

\section{B. Yamaguchi Spin Correction for Singlet-Triplet Gaps}

The singlet-triplet energy gaps obtained from the unrestricted determinant case are formally wrong, if the energy difference between the mixed (brokensymmetry) state (S1) and the triplet (T1) is taken. Therefore, we tested the effect of using the spin correction approach proposed by Yamaguchi et al!18 for the calculated gaps which is given by equation $(\mathrm{S} 2) 19$. 


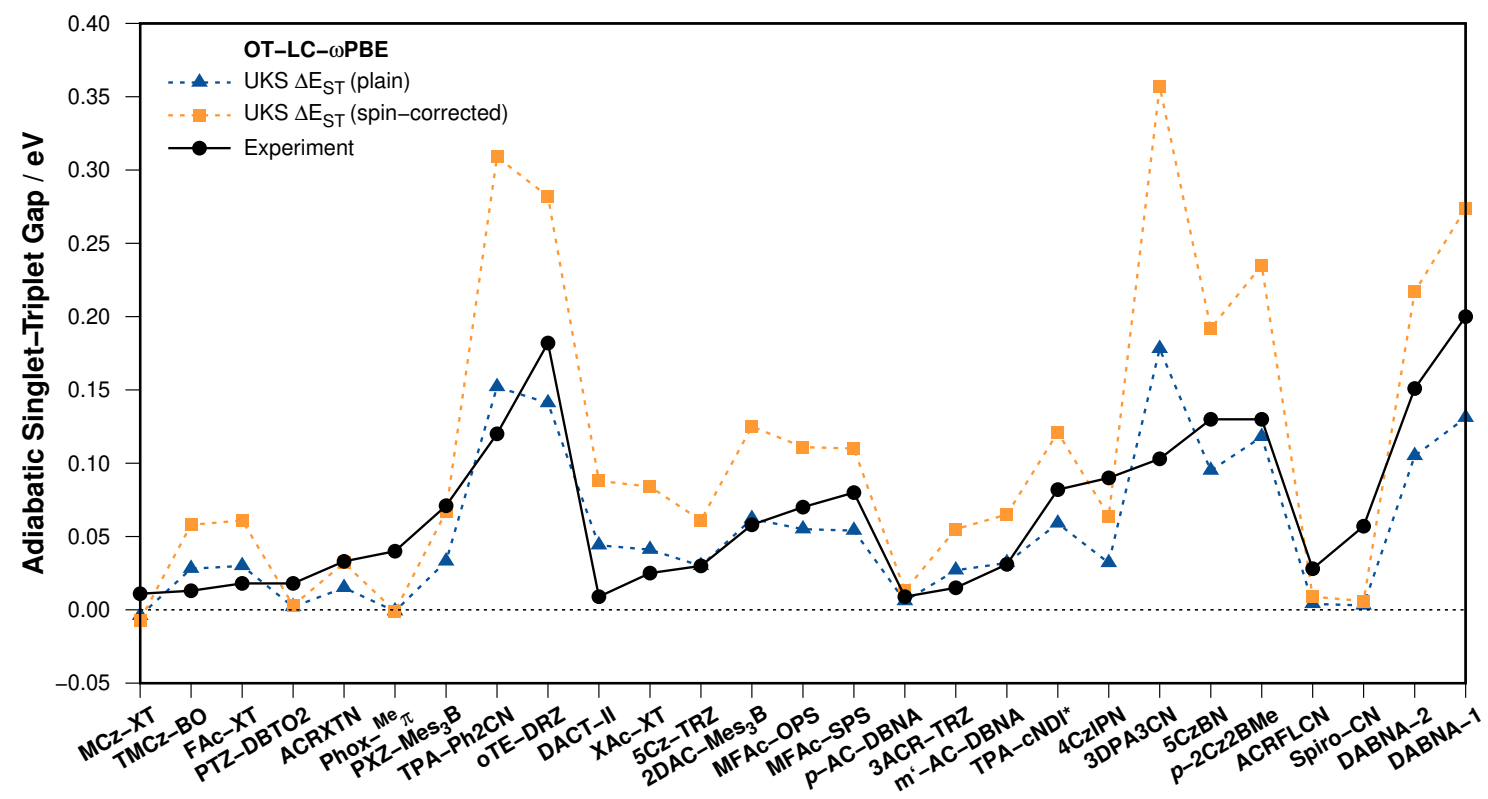

Figure S5. Adiabatic $\Delta E_{\mathrm{ST}}$ calculated with the OT-LC- $\omega$ PBE-D3 functional at the UKS level, gaps are shown as plain energy difference between states (orange) and corrected via Yamaguchi spin correction, all values in $\mathrm{eV}$.

$$
\Delta E_{\mathrm{proj}}=E_{\mathrm{S}}-E_{\mathrm{T}}=\frac{\left\langle S_{\mathrm{T}}^{2}\right\rangle\left(E_{\mathrm{BS}}-E_{\mathrm{T}}\right)}{\left\langle S_{\mathrm{T}}^{2}\right\rangle-\left\langle S_{\mathrm{BS}}^{2}\right\rangle} .
$$

$E_{\mathrm{T}}, E_{\mathrm{S}}, E_{\mathrm{BS}}$ represent the energy of the triplet, singlet and broken-symmetry solutions, and $\left\langle S_{\mathrm{T}}^{2}\right\rangle$ and $\left\langle S_{\mathrm{BS}}^{2}\right\rangle$ are the expectation value of the square of the total spin operator for the triplet and broken-symmetry solutions. Yamaguchi spin-corrected and plain singlettriplet energy gaps were calculated for the OT-LC$\omega \mathrm{PBE}-\mathrm{D} 3$ functional and are compared in figure S5. For all 27 tested emitters, the $\left\langle S_{\mathrm{T}}^{2}\right\rangle \approx 2$ with the case of largest spin contamination is approximately 2.05 , the same is true for $\left\langle S_{\mathrm{BS}}^{2}\right\rangle$ where the largest observed spin contamination was 1.05. As consequence all plain singlet-triplet gaps are multiplied by a factor of roughly 2 , leading to much larger deviations for the spin-corrected $\Delta E_{\mathrm{ST}}$ compared to the plain energy differences, see figure $\mathrm{S} 5$.

\section{Statistical Measures}

- Average: $\bar{x}=\frac{1}{n} \sum_{i} x_{i}$

- Mean deviation (MD): $\mathrm{MD}=\frac{1}{n} \sum_{i}\left(\mathrm{x}_{i}-\mathrm{r}_{i}\right)$

- Mean absolute deviation (MAD): MAD = $\frac{1}{n} \sum_{i}\left|\mathrm{x}_{i}-\mathrm{r}_{i}\right|$
- Root mean square deviation (RMSD) : RMSD = $\sqrt{\frac{1}{n} \sum_{i=1}^{n} x_{i}^{2}}$

- Bessel corrected standard deviation (SD): $\mathrm{SD}=$ $\sqrt{\frac{1}{n-1} \sum_{i}\left(\mathrm{x}_{i}-\mathrm{r}_{i}-\mathrm{MD}\right)^{2}}$

- Maximum absolute deviation (AMAX): AMAX = $\max \left\{\left|\mathrm{x}_{i}-\mathrm{r}_{i}\right|\right\}$

\section{Set A: Statistical Analysis}

The following tables S6, S7 and $\mathrm{S} 8$ show the calculated adiabatic $\Delta E_{\mathrm{ST}}$ values for set $\mathrm{A}$. The data is visualized in figure 3 of the main text. The def2-SVP basis set was employed and the dielectric constant of toluene $(\varepsilon=2.37)$ for the IEF-PCM implicit equilibrium solvation model. The fixed $\omega$ values used in the calculations (FX-"Functional") are $0.175,0.155$ and 0.134 for LC$\omega \mathrm{PBE}, \omega \mathrm{B} 97 \mathrm{M}-\mathrm{V}$ and CAM-QTP(01), respectively. 
Table S6. Adiabatic $\Delta E_{\mathrm{ST}}$ obtained with CAMQTP(01), experimental gaps and statistics for set A, OT and FX refer to functionals with optimally tuned or fixed $\omega$, UKS and ROKS were denoted $\mathrm{U}$ and $\mathrm{RO}$, all values in $\mathrm{eV}$.

\begin{tabular}{llrrrrr}
\hline$\#$ & System & $\mathrm{OT} / \mathrm{U}$ & $\mathrm{OT} / \mathrm{RO}$ & $\mathrm{FX} / \mathrm{U}$ & $\mathrm{FX} / \mathrm{RO}$ & Exp. \\
\hline 4 & PTZ-DBTO2 & 0.001 & 0.006 & 0.002 & 0.008 & 0.018 \\
5 & ACRXTN & 0.016 & 0.046 & 0.023 & 0.053 & 0.033 \\
7 & PXZ-Mes 3 B & 0.042 & 0.057 & 0.041 & 0.056 & 0.071 \\
9 & oTE-DRZ & 0.160 & 0.145 & 0.152 & 0.143 & 0.182 \\
10 & DACT-II & 0.058 & 0.043 & 0.058 & 0.042 & 0.009 \\
14 & MFAc-OPS & 0.052 & 0.082 & 0.046 & 0.077 & 0.070 \\
15 & MFAc-SPS & 0.065 & 0.101 & 0.055 & 0.092 & 0.080 \\
17 & 3ACR-TRZ & 0.028 & 0.061 & 0.022 & 0.054 & 0.015 \\
20 & 4 CzIPN & 0.047 & 0.063 & 0.056 & 0.069 & 0.090 \\
21 & 3DPA3CN & 0.187 & 0.127 & 0.203 & 0.124 & 0.103 \\
22 & 5 CzBN & 0.151 & 0.160 & 0.162 & 0.171 & 0.130 \\
24 & ACRFLCN & 0.004 & 0.012 & 0.004 & 0.006 & 0.028 \\
25 & Spiro-CN & 0.002 & -0.001 & 0.002 & 0.000 & 0.057 \\
26 & DABNA-2 & 0.106 & 0.213 & 0.115 & 0.220 & 0.151 \\
27 & DABNA-1 & 0.133 & 0.219 & 0.133 & 0.219 & 0.200 \\
\hline MD & -0.012 & 0.006 & -0.011 & 0.006 & - \\
MAD & 0.035 & 0.028 & 0.036 & 0.028 & - \\
AMIN & 0.013 & 0.012 & 0.007 & 0.007 & - \\
AMAX & 0.084 & 0.062 & 0.100 & 0.069 & - \\
SD & 0.040 & 0.033 & 0.043 & 0.034 & - \\
\hline
\end{tabular}

Table S7. Adiabatic $\Delta E_{\mathrm{ST}}$ obtained with $\omega \mathbf{B 9 7 M}-\mathbf{V}$, experimental gaps and statistics for set A, OT and FX refer to functionals with optimally tuned or fixed $\omega$, UKS and ROKS were denoted $\mathrm{U}$ and $\mathrm{RO}$, all values in $\mathrm{eV}$.

\begin{tabular}{llrrrrr}
\hline \# & System & OT/U & OT/RO & FX/U & FX/RO & Exp. \\
\hline 4 & PTZ-DBTO2 & 0.002 & 0.008 & 0.003 & 0.009 & 0.018 \\
5 & ACRXTN & 0.014 & 0.036 & 0.021 & 0.043 & 0.033 \\
7 & PXZ-Mes 3 B & 0.029 & 0.040 & 0.031 & 0.041 & 0.071 \\
9 & oTE-DRZ & 0.142 & 0.126 & 0.133 & 0.124 & 0.182 \\
10 & DACT-II & 0.048 & 0.032 & 0.048 & 0.032 & 0.009 \\
14 & MFAc-OPS & 0.040 & 0.066 & 0.034 & 0.061 & 0.070 \\
15 & MFAc-SPS & 0.036 & 0.076 & 0.047 & 0.078 & 0.080 \\
17 & 3ACR-TRZ & 0.030 & 0.049 & 0.022 & 0.043 & 0.015 \\
20 & 4CzIPN & 0.034 & 0.052 & 0.038 & 0.055 & 0.090 \\
21 & 3DPA3CN & 0.184 & 0.108 & 0.203 & 0.106 & 0.103 \\
22 & 5 CzBN & 0.095 & 0.106 & 0.109 & 0.115 & 0.130 \\
24 & ACRFLCN & 0.005 & 0.008 & 0.005 & 0.008 & 0.028 \\
25 & Spiro-CN & 0.002 & 0.000 & 0.002 & 0.000 & 0.057 \\
26 & DABNA-2 & 0.126 & 0.189 & 0.139 & 0.199 & 0.151 \\
27 & DABNA-1 & 0.158 & 0.201 & 0.157 & 0.201 & 0.200 \\
\hline MD & -0.019 & -0.009 & -0.016 & -0.008 & - \\
MAD & 0.037 & 0.023 & 0.036 & 0.023 & - \\
AMIN & 0.015 & 0.001 & 0.007 & 0.001 & - \\
AMAX & 0.081 & 0.057 & 0.100 & 0.058 & - \\
SD & 0.038 & 0.029 & 0.040 & 0.029 & - \\
\hline & & & & & \\
& & & & & -
\end{tabular}


Table S8. Adiabatic $\Delta E_{\mathrm{ST}}$ obtained with LC- $\omega$ PBE-D3, experimental gaps and statistics for set A, OT and FX refer to functionals with optimally tuned or fixed $\omega$, UKS and ROKS were denoted $\mathrm{U}$ and $\mathrm{RO}$, all values in $\mathrm{eV}$.

\begin{tabular}{llrrrrr}
\hline \# & System & $\mathrm{OT} / \mathrm{U}$ & $\mathrm{OT} / \mathrm{RO}$ & $\mathrm{FX} / \mathrm{U}$ & $\mathrm{FX} / \mathrm{RO}$ & $\mathrm{Exp}$. \\
\hline 4 & PTZ-DBTO2 & 0.002 & 0.005 & 0.003 & 0.007 & 0.018 \\
5 & ACRXTN & 0.015 & 0.038 & 0.027 & 0.048 & 0.033 \\
7 & PXZ-Mes ${ }_{3} \mathrm{~B}$ & 0.033 & 0.038 & 0.035 & 0.039 & 0.071 \\
9 & oTE-DRZ & 0.141 & 0.120 & 0.131 & 0.116 & 0.182 \\
10 & DACT-II & 0.044 & 0.028 & 0.044 & 0.028 & 0.009 \\
14 & MFAc-OPS & 0.055 & 0.077 & 0.049 & 0.070 & 0.070 \\
15 & MFAc-SPS & 0.054 & 0.077 & 0.046 & 0.069 & 0.080 \\
17 & 3ACR-TRZ & 0.027 & 0.046 & 0.019 & 0.040 & 0.015 \\
20 & 4CzIPN & 0.032 & 0.048 & 0.036 & 0.051 & 0.090 \\
21 & 3DPA3CN & 0.178 & 0.108 & 0.195 & 0.107 & 0.103 \\
22 & 5 CzBN & 0.095 & 0.102 & 0.109 & 0.110 & 0.130 \\
24 & ACRFLCN & 0.004 & 0.007 & 0.004 & 0.007 & 0.028 \\
25 & Spiro-CN & 0.003 & -0.007 & 0.000 & -0.003 & 0.057 \\
26 & DABNA-2 & 0.105 & 0.191 & 0.117 & 0.206 & 0.151 \\
27 & DABNA-1 & 0.131 & 0.208 & 0.131 & 0.208 & 0.200 \\
\hline & MD & -0.021 & -0.010 & -0.019 & -0.009 & - \\
MAD & 0.037 & 0.025 & 0.037 & 0.026 & - \\
AMIN & 0.012 & 0.003 & 0.004 & 0.000 & - \\
AMAX & 0.075 & 0.064 & 0.092 & 0.066 & - \\
SD & 0.038 & 0.031 & 0.040 & 0.032 & - \\
\hline
\end{tabular}


Full Set: Statistical Analysis

The following tables S9, S10, S11 and S12 show the calculated adiabatic $\Delta E_{\mathrm{ST}}$ values for the whole benchmark set for the $\omega \mathrm{B} 97 \mathrm{M}-\mathrm{V}, \mathrm{LC}-\omega \mathrm{PBE}$ and PBE0-D4 functionals and the dipole moments of each singlet and triplet state calculated with each functional for the whole benchmark set.

Table S9. Calculated adiabatic $\Delta E_{\mathrm{ST}}$ obtained with the $\omega \mathbf{B 9 7 M - V}, \mathbf{L C}-\omega \mathbf{P B E}-\mathbf{D} 3$ and PBE0-D4 functional, compared to experimental gaps and statistical analysis for the whole benchmark set (set A + set B), UKS and ROKS were denoted $\mathrm{U}$ and $\mathrm{RO}$ respectively, all values in $\mathrm{eV}$.

\begin{tabular}{|c|c|c|c|c|c|c|c|}
\hline \multirow[b]{2}{*}{$\#$} & \multicolumn{2}{|c|}{$\omega \mathbf{B 9 7 M}$-V } & \multicolumn{2}{|c|}{ LC- $\omega$ PBE-D3 } & \multicolumn{2}{|c|}{ PBE0-D4 } & \multirow[b]{2}{*}{ Exp. } \\
\hline & $\mathrm{U}$ & $\mathrm{RO}$ & $\mathrm{U}$ & $\mathrm{RO}$ & $\mathrm{U}$ & $\mathrm{RO}$ & \\
\hline 1 & 0.000 & 0.011 & -0.004 & 0.004 & 0.021 & 0.051 & 0.011 \\
\hline 2 & 0.007 & 0.052 & 0.028 & 0.073 & 0.054 & 0.139 & 0.013 \\
\hline 3 & 0.027 & 0.053 & 0.030 & 0.053 & 0.061 & 0.092 & 0.018 \\
\hline 4 & 0.002 & 0.008 & 0.002 & 0.005 & 0.0 & 0.072 & 0.018 \\
\hline 5 & 0.014 & 0.036 & 0.015 & 0.038 & 0.042 & 0.076 & 0.033 \\
\hline 6 & 0.000 & 0.006 & -0.001 & -0.015 & -0.001 & 0.003 & 0.040 \\
\hline 7 & 0.029 & 0.040 & 0.033 & 0.038 & 0.066 & 0.097 & 0.071 \\
\hline 8 & 0.164 & 0.108 & 0.152 & 0.106 & & & 0.120 \\
\hline 9 & 0.142 & 0.126 & 0.141 & 0.120 & 0.1 & 0.149 & 0.182 \\
\hline 10 & 0.048 & 0.032 & 0.044 & 0.028 & & 0.056 & 0.009 \\
\hline 11 & 0.037 & 0.065 & 0.041 & 0.065 & 0.0 & 0.101 & 0.025 \\
\hline 12 & 0.022 & 0.032 & 0.030 & 0.041 & & & 0.030 \\
\hline 13 & 0.063 & 0.056 & 0.062 & 0.044 & 0.060 & 0.079 & 0.058 \\
\hline 14 & 0.040 & 0.066 & 0.055 & 0.077 & 0.055 & 0.090 & 0.070 \\
\hline 15 & 0.036 & 0.076 & 0.054 & 0.077 & 0.0 & 0.117 & 70.080 \\
\hline 16 & & 0.022 & & & & & 09 \\
\hline 17 & 0.030 & 0.049 & 0.027 & 0.046 & 0.046 & 0.076 & 0.015 \\
\hline 18 & 0.020 & 0.031 & 0.032 & 0.038 & 0.030 & 0.001 & 0.031 \\
\hline 19 & 0.083 & 0.070 & 0.059 & 0.055 & 0.088 & 0.128 & 0.082 \\
\hline 20 & & & & & & & 0.090 \\
\hline 21 & 0.184 & 0.108 & 0.178 & 0.108 & 0.124 & 0.146 & 0.103 \\
\hline 22 & 0.095 & 0.106 & 0.095 & 0.102 & 0.098 & 0.109 & 0.130 \\
\hline 23 & 0.138 & 0.187 & 0.118 & 0.174 & 0.135 & 0.204 & 0.130 \\
\hline 24 & 0.005 & 0.008 & 0.004 & 0.007 & & 0.030 & 0.028 \\
\hline 25 & 0.002 & 0.000 & 0.003 & -0.007 & 0.002 & 0.008 & 0.057 \\
\hline 26 & 0.126 & 0.189 & 0.105 & 0.191 & 0.060 & 0.203 & 0.151 \\
\hline 27 & 0.158 & 0.201 & 0.131 & 0.208 & 0.077 & 0.227 & 0.200 \\
\hline $\mathrm{MD}$ & -0.011 & -0.001 & -0.012 & -0.002 & -0.007 & 0.026 & 5 - \\
\hline MAD & 0.027 & 0.022 & 0.027 & 0.026 & 0.029 & 0.042 & - \\
\hline AMIN & 0.001 & 0.000 & 0.000 & 0.003 & 0.001 & 0.002 & - \\
\hline & 0.081 & 0.057 & 0.075 & 0.064 & 0.123 & 0.126 & - \\
\hline $\mathrm{SD}$ & 0.032 & 0.029 & 0.032 & 0.033 & 0.041 & 0.042 & - \\
\hline
\end{tabular}

Full Set: Excited State Dipole Moments

Table S10. Dipole moments of excited states calculated with the $\omega \mathbf{B 9 7} \mathbf{M}-\mathbf{V}$ functional and the UKS and ROKS method, note that the T1 ROKS results are from single points on the T1 UKS optimized geometry, all values in Debye.

\begin{tabular}{llrrrrr}
\hline \# & System & $\mathrm{S} 0$ & $\mathrm{U}(\mathrm{S} 1)$ & $\mathrm{RO}(\mathrm{S} 1)$ & $\mathrm{U}(\mathrm{T} 1)$ & $\mathrm{RO}(\mathrm{T} 1)$ \\
\hline 1 & MCz-XT & 3.1 & 21.0 & 21.0 & 21.0 & 20.9 \\
2 & TMCz-BO & 3.9 & 21.0 & 21.0 & 0.5 & 0.6 \\
3 & FAc-XT & 2.9 & 22.1 & 22.2 & 18.1 & 17.8 \\
4 & PTZ-DBTO2 & 3.8 & 23.0 & 23.3 & 23.0 & 23.1 \\
5 & ACRXTN & 3.0 & 21.6 & 21.6 & 18.9 & 18.7 \\
6 & Phox- ${ }^{M e} \pi$ & 3.5 & 23.2 & 23.3 & 23.1 & 23.2 \\
7 & PXZ-Mes 3 B & 4.1 & 21.0 & 20.6 & 17.5 & 17.3 \\
8 & TPA-Ph2CN & 1.9 & 24.7 & 19.4 & 18.0 & 17.8 \\
9 & oTE-DRZ & 1.8 & 11.6 & 11.2 & 10.2 & 10.2 \\
10 DACT-II & 2.7 & 31.6 & 31.5 & 28.5 & 28.2 \\
11 & XAc-XT & 2.9 & 20.8 & 20.9 & 16.3 & 16.0 \\
12 & 5Cz-TRZ & 1.5 & 12.4 & 12.8 & 11.5 & 11.4 \\
13 & 2DAC-Mes 3 B & 4.2 & 28.6 & 28.1 & 22.7 & 22.3 \\
14 & MFAc-OPS & 4.3 & 20.9 & 21.1 & 17.1 & 17.0 \\
15 & MFAc-SPS & 5.6 & 19.5 & 20.7 & 15.8 & 15.5 \\
16 & p-AC-DBNA & 0.5 & 25.3 & 25.4 & 23.3 & 23.0 \\
17 3ACR-TRZ & 0.1 & 27.2 & 27.3 & 22.5 & 22.1 \\
18 m'-AC-DBNA & 1.1 & 20.4 & 20.6 & 17.9 & 17.6 \\
19 & TPA-cNDI* & 0.9 & 30.8 & 29.8 & 26.0 & 25.7 \\
20 & 4CzIPN & 5.4 & 16.7 & 16.9 & 16.4 & 16.3 \\
21 & 3DPA3CN & 0.5 & 21.7 & 20.6 & 17.9 & 17.8 \\
22 & 5CzBN & 4.3 & 13.9 & 14.1 & 11.2 & 11.3 \\
23 & p-2Cz2BMe & 0.1 & 0.0 & 0.0 & 0.0 & 0.0 \\
24 & ACRFLCN & 1.1 & 14.8 & 14.8 & 14.8 & 14.7 \\
25 & Spiro-CN & 2.0 & 10.8 & 10.8 & 10.7 & 10.7 \\
26 & DABNA-2 & 2.7 & 8.3 & 7.9 & 7.5 & 7.4 \\
27 & DABNA-1 & 2.7 & 7.4 & 6.5 & 6.5 & 6.4 \\
\hline & & & & & &
\end{tabular}


Table S11. Dipole moments of excited states calculated with the LC- $\omega$ PBE-D3 functional and the UKS and ROKS method, note that the T1 ROKS results are from single points on the T1 UKS optimized geometry, all values in Debye.

\begin{tabular}{|c|c|c|c|c|c|c|}
\hline$\#$ & System & S0 & $\mathrm{U}(\mathrm{S} 1)$ & $\mathrm{RO}(\mathrm{S} 1)$ & $\mathrm{U}(\mathrm{T} 1)$ & $\mathrm{RO}(\mathrm{T} 1)$ \\
\hline 1 & $\mathrm{MCz}-\mathrm{XT}$ & 3.1 & 20.9 & 20.9 & 20.9 & 20.8 \\
\hline 2 & TMCz-BO & 3.8 & 21.2 & 21.1 & 0.5 & 0.6 \\
\hline 3 & FAc-XT & 2.9 & 21.9 & 22.0 & 17.6 & 17.3 \\
\hline 4 & PTZ-DBTO2 & 3.5 & 22.7 & 23.0 & 22.7 & 22.8 \\
\hline 5 & ACRXTN & 3.0 & 21.5 & 21.6 & 18.4 & 18.1 \\
\hline 6 & Phox- ${ }^{M e} \pi$ & 3.5 & 23.2 & 23.3 & 23.2 & 23.3 \\
\hline 7 & $\mathrm{PXZ}^{-\mathrm{Mes}_{3} \mathrm{~B}}$ & 4.2 & 21.2 & 20.6 & 17.3 & 17.1 \\
\hline 8 & TPA-Ph2CN & 1.7 & 25.1 & 20.5 & 18.3 & 18.1 \\
\hline 9 & oTE-DRZ & 1.8 & 11.2 & 10.9 & 10.1 & 10.1 \\
\hline 10 & DACT-II & 2.7 & 32.0 & 31.5 & 29.2 & 28.9 \\
\hline 11 & $\mathrm{XAc-XT}$ & 2.9 & 20.7 & 20.8 & 15.7 & 15.4 \\
\hline 12 & $5 \mathrm{Cz}-\mathrm{TRZ}$ & 1.4 & 9.2 & 9.6 & 8.8 & 8.7 \\
\hline 13 & $2 \mathrm{DAC}-\mathrm{Mes}_{3} \mathrm{~B}$ & 4.4 & 28.6 & 28.0 & 22.8 & 22.4 \\
\hline 14 & MFAc-OPS & 4.4 & 21.1 & 21.4 & 16.1 & 15.9 \\
\hline 15 & MFAc-SPS & 5.6 & 20.6 & 20.9 & 15.2 & 15.0 \\
\hline 16 & $p$-AC-DBNA & 0.8 & 25.4 & 25.4 & 22.7 & 22.3 \\
\hline 17 & 3ACR-TRZ & 0.1 & 27.3 & 27.3 & 22.6 & 22.2 \\
\hline 18 & m'-AC-DBNA & 1.1 & 20.6 & 20.7 & 17.6 & 17.1 \\
\hline 19 & TPA-cNDI* & 0.7 & 29.3 & 29.4 & 26.3 & 26.0 \\
\hline 20 & $4 \mathrm{CzIPN}$ & 5.2 & 16.6 & 16.8 & 16.3 & 16.1 \\
\hline 21 & 3DPA3CN & 0.4 & 21.6 & 20.9 & 17.9 & 17.9 \\
\hline 22 & $5 \mathrm{CzBN}$ & 4.2 & 13.7 & 14.1 & 12.3 & 12.4 \\
\hline 23 & $p-2 \mathrm{Cz} 2 \mathrm{BMe}$ & 0.2 & 0.6 & 4.5 & 0.0 & 0.0 \\
\hline 24 & ACRFLCN & 1.2 & 14.6 & 14.5 & 14.6 & 14.4 \\
\hline 25 & Spiro-CN & 2.0 & 10.3 & 10.2 & 10.2 & 10.2 \\
\hline 26 & DABNA-2 & 2.8 & 8.1 & 8.4 & 7.5 & 7.3 \\
\hline 27 & DABNA-1 & 2.9 & 7.4 & 6.8 & 6.6 & 6.5 \\
\hline
\end{tabular}

Table S12. Dipole moments of excited states calculated with the PBE0-D4 functional and the UKS and ROKS method, note that the T1 ROKS results are from single points on the T1 UKS optimized geometry, all values in Debye.

\begin{tabular}{|c|c|c|c|c|c|c|}
\hline$\#$ & System & S0 & $\mathrm{U}(\mathrm{S} 1)$ & $\mathrm{RO}(\mathrm{S} 1)$ & $\mathrm{U}(\mathrm{T} 1)$ & $\mathrm{RO}(\mathrm{T} 1)$ \\
\hline 1 & $\mathrm{MCz}-\mathrm{XT}$ & 3.2 & 21.5 & 21.6 & 21.6 & 16.4 \\
\hline 2 & TMCz-BO & 3.9 & 14.3 & 19.9 & 19.9 & 7.5 \\
\hline 3 & FAc-XT & 3.0 & 22.3 & 22.4 & 22.4 & 16.5 \\
\hline 4 & PTZ-DBTO2 & 3.9 & 20.5 & 21.8 & 21.8 & 15.7 \\
\hline 5 & ACRXTN & 3.1 & 21.7 & 22.0 & 22.0 & 17.2 \\
\hline 6 & $\mathrm{Phox}^{M e} \pi$ & 3.5 & 22.9 & 23.1 & 23.1 & 22.9 \\
\hline 7 & $\mathrm{PXZ}^{-\mathrm{Mes}_{3} \mathrm{~B}}$ & 4.2 & 18.7 & 19.4 & 19.4 & 14.0 \\
\hline 8 & TPA-Ph2CN & 2.1 & 22.0 & 19.0 & 19.0 & 17.6 \\
\hline 9 & oTE-DRZ & 1.8 & 11.0 & 11.0 & 11.0 & 9.7 \\
\hline 10 & DACT-II & 2.4 & 31.0 & 31.4 & 31.4 & 27.6 \\
\hline 11 & $\mathrm{XAc}-\mathrm{XT}$ & 3.0 & 21.0 & 21.1 & 21.1 & 15.2 \\
\hline 12 & $5 \mathrm{Cz}-\mathrm{TRZ}$ & 1.4 & 3.6 & 4.4 & 4.4 & 5.5 \\
\hline 13 & 2DAC-Mes 3 B & 4.1 & 26.7 & 27.6 & 27.6 & 21.1 \\
\hline 14 & MFAc-OPS & 4.5 & 21.2 & 21.7 & 21.7 & 16.0 \\
\hline 15 & MFAc-SPS & 5.8 & 19.7 & 20.7 & 20.7 & 13.6 \\
\hline 16 & $p$-AC-DBNA & 0.6 & 8.4 & 8.3 & 8.3 & 19.7 \\
\hline 17 & 3ACR-TRZ & 0.1 & 27.4 & 19.3 & 19.3 & 21.1 \\
\hline 18 & m'-AC-DBNA & 1.2 & 4.3 & 4.3 & 4.3 & 16.5 \\
\hline 19 & TPA-cNDI* & 1.2 & 4.3 & 5.2 & 5.2 & 4.7 \\
\hline 20 & $4 \mathrm{CzIPN}$ & 5.4 & 15.8 & 15.9 & 15.9 & 15.0 \\
\hline 21 & 3DPA3CN & 0.6 & 19.8 & 20.5 & 20.5 & 17.3 \\
\hline 22 & $5 \mathrm{CzBN}$ & 4.4 & 5.1 & 5.3 & 5.3 & 8.5 \\
\hline 23 & $p-2 \mathrm{Cz} 2 \mathrm{BMe}$ & 0.1 & 0.0 & 0.1 & 0.1 & 0.0 \\
\hline 24 & ACRFLCN & 1.1 & 14.5 & 14.4 & 14.4 & 2.5 \\
\hline 25 & Spiro-CN & 1.8 & 10.5 & 10.4 & 10.4 & 9.8 \\
\hline 26 & DABNA-2 & 2.7 & 7.9 & 9.5 & 9.5 & 7.8 \\
\hline 27 & DABNA-1 & 2.8 & 6.8 & 6.6 & 6.6 & 6.2 \\
\hline
\end{tabular}




\section{Input Files for Q-Chem}

\section{ROKS/GDM}

Example input file for a RKS ground-state single point as input for a ROKS/GDM geometry optimization for a singlet excited state with FX-LC- $\omega$ PBED3(BJ) ${ }^{\text {ATM }}$ functional, def2-SVP basis set, IEF-PCM solvation model with solvent parameters for toluene and standard integration grid. Note that thresh should be set explicitly since for orbital gradient-based methods like GDM the default thresholds in Q-Chem are not tight enough and sometimes lead to problems in the ROKS SCF.

\begin{tabular}{|c|c|}
\hline \$rem & \\
\hline thresh & 11 \\
\hline threads & 8 \\
\hline jobtype & $\mathrm{sp}$ \\
\hline dft_d & d3_bj \\
\hline dft_d3_3body & true \\
\hline method & LC-wPBE08 \\
\hline omega & 175 \\
\hline basis & def2-SVP \\
\hline gen_scfman & true \\
\hline scf_algorithm & diis \\
\hline scf_convergence & 8 \\
\hline mem_static & 8000 \\
\hline mem_total & 20000 \\
\hline sym_ignore & true \\
\hline solvent_method & $\mathrm{pcm}$ \\
\hline \$end & \\
\hline$\$ p c m$ & \\
\hline theory & iefpcm \\
\hline \$end & \\
\hline \$solvent & \\
\hline dielectric & 2.3741 \\
\hline dielectric_infi & 2.2407 \\
\hline \$end & \\
\hline \$molecule & \\
\hline read & input.xyz \\
\hline \$end & \\
\hline @@ & \\
\hline \$rem & \\
\hline thresh & 11 \\
\hline threads & 16 \\
\hline jobtype & opt \\
\hline$d f t \_d$ & d3_bj \\
\hline dft_d3_3body & true \\
\hline method & LC-wPBE08 \\
\hline omega & 175 \\
\hline
\end{tabular}

basis

gen_scfman

scf_guess

scf_algorithm

scf_convergence

scf_max_cycles

unrestricted

roks

mem_static

mem_total

sym_ignore

solvent_method

\$end

$\$ p c m$

theory

\$end

\$solvent

dielectric

dielectric_infi

\$end

\$molecule

01

read

\$end

ad
def2-SVP

true

read

diis_gdm

5

250

false

true

8000

20000

true

$\mathrm{pcm}$

iefpcm

2. 3741

2. 2407 


\section{UKS/IMOM}

Example input file for: a UKS ground-state single point as input for a UKS/IMOM geometry optimization for a singlet excited state with FX- $\omega$ B97M-V functional, def2-SVP basis set, IEF-PCM solvation model with solvent parameters for toluene, standard integration grid and for a system with 202 electrons (Example: TPA-Ph2CN).

\begin{tabular}{|c|c|}
\hline$\$ r e m$ & \\
\hline thresh & 11 \\
\hline threads & 8 \\
\hline jobtype & $\mathrm{sp}$ \\
\hline method & wB97M-V \\
\hline omega & 155 \\
\hline basis & $\operatorname{def} 2-S V P$ \\
\hline scf_algorithm & diis \\
\hline scf_convergence & 8 \\
\hline scf_max_cycles & 250 \\
\hline unrestricted & true \\
\hline mem_static & 8000 \\
\hline mem_total & 20000 \\
\hline sym_ignore & true \\
\hline $\begin{array}{l}\text { solvent_method } \\
\text { \$end }\end{array}$ & $\mathrm{pcm}$ \\
\hline$\$ p c m$ & \\
\hline theory & iefpcm \\
\hline \$end & \\
\hline \$solvent & \\
\hline dielectric & 2.3741 \\
\hline $\begin{array}{l}\text { dielectric_infi } \\
\text { \$end }\end{array}$ & 2.2407 \\
\hline \$molecule & \\
\hline read & input.xyz \\
\hline \$end & \\
\hline @Q & \\
\hline$\$ r e m$ & \\
\hline thresh & 11 \\
\hline threads & 8 \\
\hline method & wB97M-V \\
\hline omega & 155 \\
\hline jobtype & opt \\
\hline basis & def2-SVP \\
\hline scf_guess & read \\
\hline scf_algorithm & diis \\
\hline scf_convergence & 8 \\
\hline scf_max_cycles & 250 \\
\hline unrestricted & true \\
\hline mom_method & imom \\
\hline mom_start & 1 \\
\hline
\end{tabular}

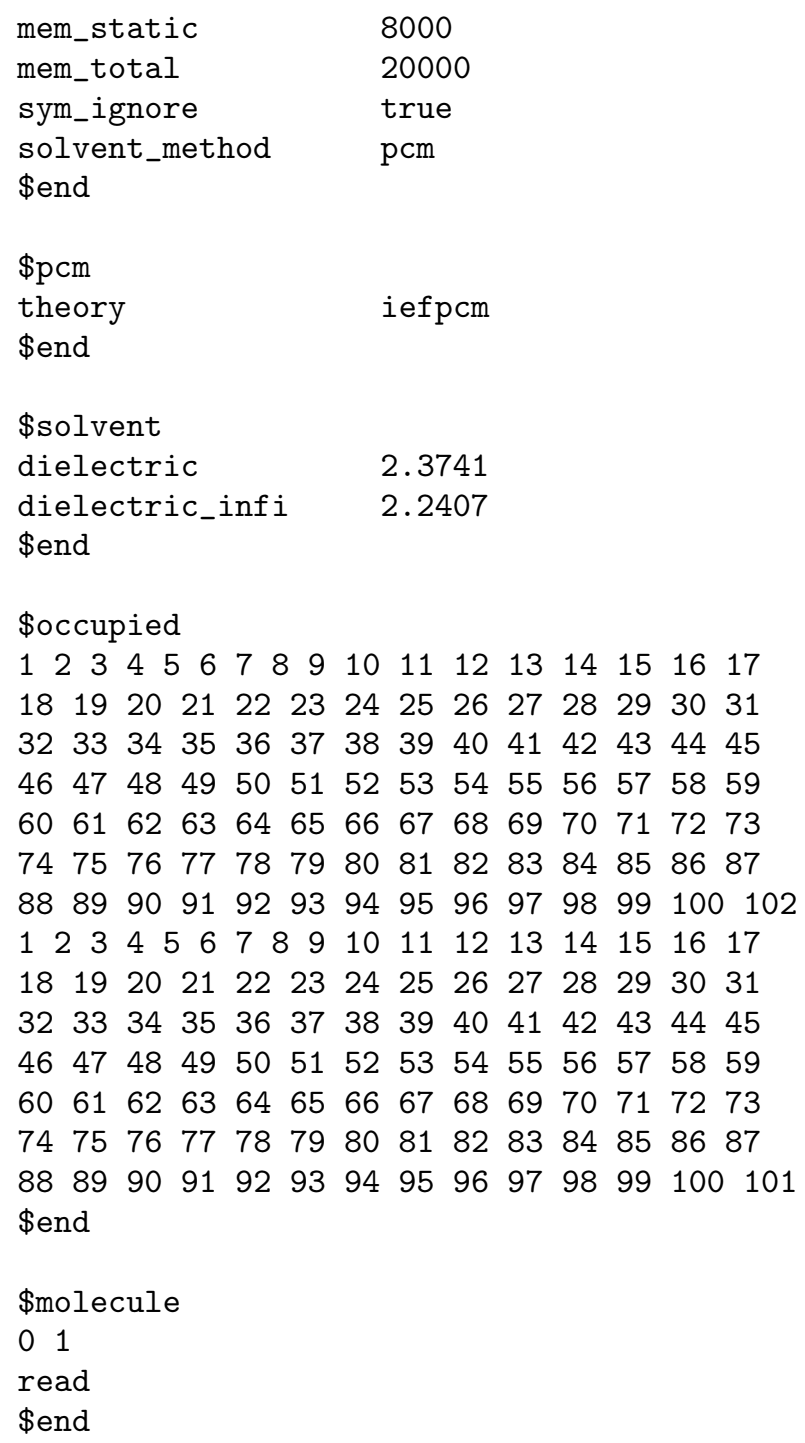

\section{Geometries}

All optimized singlet and triplet excited-state geometries calculated with either ROKS or UKS for the whole benchmark set are provided in the supplementary information in zip-file format. The optimized ground-state geometries obtained with PBEh-3c which served as input for excited-state optimizations are also provided. 


\section{REFERENCES}

(1) Shao, Y.; Gan, Z.; Epifanovsky, E.; Gilbert, A. T.; Wormit, M.; Kussmann, J.; Lange, A. W.; Behn, A.; Deng, J.; Feng, X., et al. Advances in molecular quantum chemistry contained in the Q-Chem 4 program package. Mol. Phys. 2015, 113, 184-215.

(2) Weigend, F.; Ahlrichs, R. Balanced basis sets of split valence, triple zeta valence and quadruple zeta valence quality for H to Rn: Design and assessment of accuracy. Phys. Chem. Chem. Phys. 2005, 7, 3297-3305.

(3) Cances, E.; Mennucci, B.; Tomasi, J. A new integral equation formalism for the polarizable continuum model: Theoretical background and applications to isotropic and anisotropic dielectrics. J. Chem. Phys. 1997, 10\%, 3032-3041.

(4) Jin, Y.; Bartlett, R. J. The QTP family of consistent functionals and potentials in Kohn-Sham density functional theory. J. Chem. Phys. 2016, 034107.

(5) Rohrdanz, M. A.; Herbert, J. M. Simultaneous benchmarking of ground- and excited-state properties with long-range-corrected density functional theory. $J$. Chem. Phys. 2008, 129, 034107.

(6) Rohrdanz, M. A.; Martins, K. M.; Herbert, J. M. A long-range-corrected density functional that performs well for both ground-state properties and timedependent density functional theory excitation energies, including charge-transfer excited states. J. Chem. Phys. 2009, 130, 054112.

(7) Weintraub, E.; Henderson, T. M.; Scuseria, G. E. Longrange-corrected hybrids based on a new model exchange hole. J. Chem. Theory Comput. 2009, 754-762.

(8) Mardirossian, N.; Head-Gordon, M. $\omega$ B97M-V: A combinatorially optimized, range-separated hybrid, metaGGA density functional with VV10 nonlocal correlation. J. Chem. Phys. 2016, 144, 214110.

(9) Perdew, J. P.; Burke, K.; Ernzerhof, M. Generalized gradient approximation made simple. Phys. Rev. Lett. 1996, 7r, 3865-3868, erratum Phys. Rev. Lett. 78, 1396 (1997).
(10) Adamo, C.; Barone, V. Toward reliable density functional methods without adjustable parameters: The PBE0 model. J. Chem. Phys. 1999, 110, 6158-6170.

(11) Caldeweyher, E.; Ehlert, S.; Hansen, A.; Neugebauer, H.; Spicher, S.; Bannwarth, C.; Grimme, S. A generally applicable atomic-charge dependent London dispersion correction. J. Chem. Phys. 2019, 150, 154122 .

(12) Caldeweyher, E.; Mewes, J.-M.; Ehlert, S.; Grimme, S. Extension and evaluation of the D4 London-dispersion model for periodic systems. Phys. Chem. Chem. Phys. 2020, 22, 8499-8512.

(13) Grimme, S.; Antony, J.; Ehrlich, S.; Krieg, H. A consistent and accurate ab initio parametrization of density functional dispersion correction (DFT-D) for the 94 elements H-Pu. J. Chem. Phys. 2010, 132, 154104.

(14) Grimme, S.; Ehrlich, S.; Goerigk, L. Effect of the damping function in dispersion corrected density functional theory. J. Comput. Chem. 2011, 32, 1456-1465.

(15) Santra, G.; Martin, J. M. Some observations on the performance of the most recent exchange-correlation functionals for the large and chemically diverse GMTKN55 benchmark. 2019, 2186, 030004.

(16) Vydrov, O. A.; Van Voorhis, T. Nonlocal van der Waals density functional: The simpler the better. J. Chem. Phys. 2010, 133, 244103.

(17) Shee, J.; Head-Gordon, M. Predicting excitation energies of twisted intramolecular charge-transfer states with the time-dependent density functional theory: Comparison with experimental measurements in the gas phase and solvents ranging from hexanes to acetonitrile. J. Chem. Theory Comput. 2020, 16, 62446255 .

(18) Yamaguchi, K.; Jensen, F.; Dorigo, A.; Houk, K. A spin correction procedure for unrestricted Hartree-Fock and Møller-Plesset wavefunctions for singlet diradicals and polyradicals. Chem. Phys. Lett. 1988, 149, 537-542.

(19) Das, A.; Muller, T.; Plasser, F.; Krisiloff, D. B.; Carter, E. A.; Lischka, H. Local electron correlation treatment in extended multireference calculations: Effect of acceptor-donor substituents on the biradical character of the polycyclic aromatic hydrocarbon heptazethrene. J. Chem. Theory Comput. 2017, 13, 26122622 . 